\title{
ZETA FUNCTIONS FOR ONE-DIMENSIONAL GENERALIZED SOLENOIDS
}

\author{
INHYEOP YI
}

\begin{abstract}
We compute zeta functions of 1-solenoids. When our 1-solenoid is nonorientable, we compute Artin-Mazur zeta function and Lefschetz zeta function of the 1-solenoid and its orientable double cover explicitly in terms of adjacency matrices and branch points. And we show that Artin-Mazur zeta function of orientable double cover is a rational function and a quotient of Artin-Mazur zeta function and Lefschetz zeta function of the 1-solenoid.
\end{abstract}

\section{Preliminary}

In the theory of dynamical systems, the study of periodic orbits has been one of main topics. An important tool for this purpose is the zeta function introduced by Artin and Mazur [1]. Although Artin-Mazur zeta function is defined only when the number of periodic orbits of period $n$ is finite for every $n \geq 1$ and this function is defined as a formal power series so that in general it does not converge, Artin-Mazur zeta function is one of the most useful invariants for topological conjugacy.

Later on, many different variations of Artin-Mazur zeta function were introduced by several authors (we refer $[2,5]$ ). One is Lefschetz zeta function or false zeta function introduced by Smale [6] using Lefschetz number instead of number of periodic points of period $n$. While Artin-Mazur zeta function gives geometric information about the periodic orbits of a dynamical system, Lefschetz zeta function counts periodic orbits 'algebraically'. Even though Lefschetz zeta function is similar to Artin-Mazur zeta function, interrelation between them is relatively unknown. The main goal of this paper is to find a relation between Artin-Mazur zeta function and Lefschetz zeta function for 1-solenoids.

Received by the editors January 2011. Revised February 25, 2011. Accepted February 26, 2011. 2000 Mathematics Subject Classification. 37C30, 37C15.

Key words and phrases. generalized solenoid, Artin-Mazur zeta function, Lefschetz zeta function, Lefschetz number.

This work was supported by the Ewha Womans University Research Grant of 2009. 
As in the case of subshifts of finite type, iteration behaviors of 1-solenoids are represented by their adjacency matrices. So they have relatively easy structure to investigate periodic properties. But contrary to subshifts of finite type which are generated by finite sets, 1-solenoids are inverse limits of finite directed graphs and connecting maps. Thus they have nontrivial periodic structures around vertices of graphs. Especially when the connecting maps do not preserve directions of graphs, small neighborhoods of vertices of graphs are twisted by connecting maps in inverse limit structure. We want to ignore geometric data of those twisted neighbors, and this is where Lefschetz zeta function is called.

In Section 2, we briefly review the definition and basic properties of 1-solenoids and their double covers. In Section 3, we modify the definitions of Lefschetz number and Lefschetz zeta function to 1-solenoids. Then we compute Artin-Mazur zeta functions and Lefschetz zeta functions of a nonorientable 1-solenoid and its corresponding orientable double cover to show that these two zeta functions are expressed through adjacency matrices and orbits of branch points. And we check that, ArtinMazur zeta function of orientable double cover is a rational function and a quotient of Artin-Mazur zeta function and Lefschetz zeta function of the 1-solenoid.

\section{1-solenoids and Their Orientable Double Covers}

We review the definition and basic properties of one-dimensional generalized solenoids of Williams. As general references for the notion of generalized solenoids and their orientable double covers, we refer to $[7,8,9,10,11]$.

One-dimensional Generalized Solenoids Let $X$ be a directed graph with vertex set $\mathcal{V}$ and edge set $\mathcal{E}$, and $f: X \rightarrow X$ a continuous map. We define some axioms which might be satisfied by $(X, f)$.

Axiom 0. (Indecomposability) $(X, f)$ is indecomposable.

Axiom 1. (Nonwandering) All points of $X$ are nonwandering under $f$.

Axiom 2. (Flattening) There is $k \geq 1$ such that for every $x \in X$ there is an open neighborhood $U$ of $x$ such that $f^{k}(U)$ is homeomorphic to $(-\epsilon, \epsilon)$.

Axiom 3. (Expansion) There is a metric $d$ compatible with the topology and positive constants $C$ and $\lambda$ with $\lambda>1$ such that for all $n>0$ and all points $x, y$ on a common edge of $X$, if $f^{n}$ maps the interval $[x, y]$ into an edge, then $d\left(f^{n} x, f^{n} y\right) \geq C \lambda^{n} d(x, y)$.

Axiom 4. (Nonfolding) $\left.f^{n}\right|_{X-\mathcal{V}}$ is locally one-to-one for every positive integer $n$. 
Axiom 5. (Markov) $f(\mathcal{V}) \subseteq \mathcal{V}$

Let $\bar{X}$ be the inverse limit space

$$
\bar{X}=X \stackrel{f}{\longleftarrow} X \stackrel{f}{\longleftarrow} \cdots=\left\{\left(x_{0}, x_{1}, x_{2}, \ldots\right) \in \prod_{0}^{\infty} X \mid f\left(x_{n+1}\right)=x_{n}\right\} .
$$

Suppose that $Y$ is a topological space. We call $Y$ a 1-dimensional generalized solenoid or 1-solenoid if there exists a directed graph $X$ and a continuous map $f: X \rightarrow X$ such that $(X, f)$ satisfies all six Axioms and $\bar{X}$ is homeomorphic to $Y$. And $(X, f)$ is called a presentation of $Y$. If we can choose the direction of each edge in $X$ so that the connection map $f: X \rightarrow X$ is orientation preserving or reversing, then we call $(X, f)$ an orientable presentation, and $Y$ an orientable solenoid. We call a point $x \in X$ a non-branch point if $x$ has an open neighborhood which is homeomorphic to an open interval, and branch point otherwise.

Remark 2.1. Axiom 0 means that $X$ cannot be split into two nonempty, closed, $f$-invariant subsets.

By a path in $X$ we mean a finite sequence $e_{1}^{s(1)} \cdots e_{n}^{s(n)}$ of edges such that, for $1 \leq i<n, s(i)= \pm 1$ represents the direction of $e_{i}$ and the terminal vertex of $e_{i}^{s(i)}$ is the initial vertex of $e_{i+1}^{s(i+1)}$. Let $\mathcal{E}^{*}$ denote the path set of $X$. Then the wrapping rule $\check{f}: \mathcal{E} \rightarrow \mathcal{E}^{*}$ associated with $f$ is given by

$$
\check{f}: e_{i} \mapsto e_{i, 1}^{s(i, 1)} \cdots e_{i, l(i)}^{s(i, l(i))},
$$

and the adjacency matrix $M=M_{X, f}$ of $(\mathcal{E}, \check{f})$ is given by

$$
M(i, j)=\text { number of copies of } e_{j}^{ \pm 1} \text { in } \check{f}\left(e_{i}\right) .
$$

Proposition $2.2([8,10])$. Suppose that $(X, f)$ is a presentation of 1-solenoids. Then $(\bar{X}, \bar{f})$ and $\left(\bar{X}, \overline{f^{n}}\right)$ are topologically conjugate for every positive integer $n$.

Therefore, for the purpose of computing zeta functions, we can replace $(X, f)$ with $\left(X, f^{n}\right)$ where $n$ is a positive integer such that for every $x \in X$ there is an open neighborhood $U_{x}$ such that $f^{n}\left(U_{x}\right)$ is an open interval by Flattening Axiom.

Standing Assumption. In this paper, we always assume that $(X, f)$ is a presentation of a 1 -solenoid such that every point $x \in X$ has a neighborhood $U_{x}$ such that $f\left(U_{x}\right)$ is an interval.

Remark 2.3. Suppose that $(X, f)$ is a presentation of 1 -solenoids. Then there is a bijection between the set of fixed points of $\left(X, f^{n}\right)$ and that of $\left(\bar{X}, \overline{f^{n}}\right)$ for every natural number $n$. 
Orientable Double Covers of 1 -solenoids Let $(X, f)$ be a presentation of a 1solenoid $\bar{X}$. Then, imitating the construction of orientable double covers of nonorientable manifolds and path lifting property of Algebraic Topology, we can construct a new directed graph $\tilde{X}$, a lifting $\tilde{f}: \tilde{X} \rightarrow \tilde{X}$ of $f: X \rightarrow X$ and a double covering map $p: \tilde{X} \rightarrow X$ such that $f \circ p=p \circ \tilde{f}$.

If $(X, f)$ is not orientable, then $(\tilde{X}, \tilde{f})$ is an orientable presentation of a 1-solenoid, and the induced map $\bar{p}: \bar{X} \rightarrow \bar{X}$ is a double covering map. The new presentation $(\tilde{X}, \tilde{f})(\bar{X}$, respectively) is called an orientable double cover of $(X, f)(\bar{X}$, respectively) [11].

Because the construction of orientable double cover is too technical, we refer to [11] for accurate description, and we mention only relevant properties and an example. See also $[3,12]$.

Proposition 2.4 ([11]). Suppose that $(X, f)$ is a presentation of a 1-solenoid. Then $(\tilde{X}, \tilde{f})$ is an orientable double cover of $(X, f)$.

(1) If $(X, f)$ is orientable, then $(\tilde{X}, \tilde{f})$ is the disjoint union of orientable presentations $\left(X_{1}, f_{1}\right)$ and $\left(X_{2}, f_{2}\right)$ of 1-solenoids such that $\left.p\right|_{X_{1}}$ is an orientationpreserving homeomorphism, $\left.p\right|_{X_{2}}$ is an orientation-reversing homeomorphism, and $\left(X_{i}, f_{i}\right)$ is topologically conjugate to $(X, f), i=1,2$.

(2) If $(X, f)$ is nonorientable, then $(\tilde{X}, \tilde{f})$ is an orientable presentation of a 1-solenoid.

Suppose that $(X, f)$ is a presentation of a nonorientable 1-solenoid with the edge set $\mathcal{E}$ and that $(\tilde{X}, \tilde{f})$ is its orientable double cover with the edge set $\tilde{\mathcal{E}}$ and the covering map $p: \tilde{X} \rightarrow X$. Let $M$ be the $n \times n$ adjacency matrix of $(\mathcal{E}, \tilde{f})$, and $\tilde{M}$ the $2 n \times 2 n$ adjacency matrix of $(\tilde{\mathcal{E}}, \tilde{\tilde{f}})$.

Proposition 2.5 ([11]). Let $M$ and $\tilde{M}$ be as above. Then there are two $n \times n$ nonnegative integer matrices $M_{1}$ and $M_{2}$ such that

$$
M=M_{1}+M_{2} \text { and } \tilde{M}=\left(\begin{array}{ll}
M_{1} & M_{2} \\
M_{2} & M_{1}
\end{array}\right) .
$$

The matrices $M_{1}$ and $M_{2}$ are given by

$$
\begin{aligned}
& M_{1}(i, j)=\text { number of copies of } e_{j} \text { in } \check{f}\left(e_{i}\right) \text { and } \\
& M_{2}(i, j)=\text { number of copies of } e_{j}^{-1} \text { in } \check{f}\left(e_{i}\right) .
\end{aligned}
$$

Example 2.6. Suppose that $X$ is Figure 1. Then its double cover $\tilde{X}$ is given by Figure 2. 


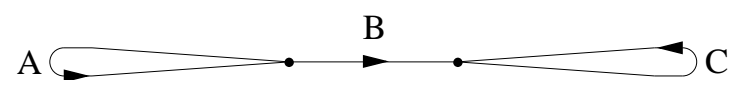

Figure 1. Directed graph $X$ for a nonorientable solenoid

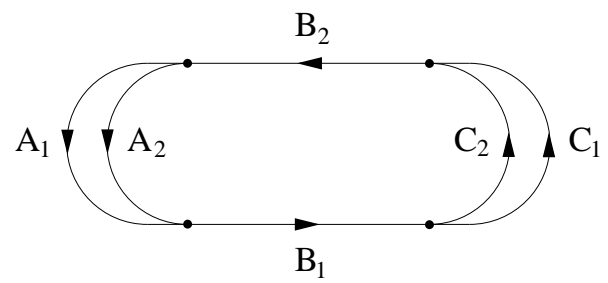

Figure 2. Double cover of $X$

When $f: X \rightarrow X$ is given by

$$
A \mapsto B^{-1} A B, B \mapsto C B^{-1} A \text {, and } C \mapsto B C B^{-1},
$$

$(X, f)$ is a presentation of a nonorientable solenoid. And the lifting $\tilde{f}: \tilde{X} \rightarrow \tilde{X}$ is given by

$$
\begin{aligned}
& A_{1} \mapsto B_{2} A_{1} B_{1}, B_{1} \mapsto C_{1} B_{2} A_{1}, C_{1} \mapsto B_{1} C_{1} B_{2} \text { and } \\
& A_{2} \mapsto B_{2} A_{2} B_{1}, B_{2} \mapsto A_{2} B_{1} C_{2}, C_{2} \mapsto B_{1} C_{2} B_{2} .
\end{aligned}
$$

So we have

$$
M_{1}=\left[\begin{array}{lll}
1 & 1 & 0 \\
1 & 0 & 1 \\
0 & 1 & 1
\end{array}\right], M_{2}=\left[\begin{array}{lll}
0 & 1 & 0 \\
0 & 1 & 0 \\
0 & 1 & 0
\end{array}\right], M=\left[\begin{array}{lll}
1 & 2 & 0 \\
1 & 1 & 1 \\
0 & 2 & 1
\end{array}\right]
$$

and

$$
\tilde{M}=\left[\begin{array}{llllll}
1 & 1 & 0 & 0 & 1 & 0 \\
1 & 0 & 1 & 0 & 1 & 0 \\
0 & 1 & 1 & 0 & 1 & 0 \\
0 & 1 & 0 & 1 & 1 & 0 \\
0 & 1 & 0 & 1 & 0 & 1 \\
0 & 1 & 0 & 0 & 1 & 1
\end{array}\right]
$$

\section{Zeta Functions}

We compute Artin-Mazur zeta functions and Lefschetz zeta functions for nonorientable 1-solenoids and their orientable double coverings.

Definition $3.1([1,6])$. Suppose that $Y$ is a set and that $g: Y \rightarrow Y$ is a function with the property that $N_{m}<\infty$ for every $m=1,2, \ldots$ where $N_{m}=N_{m}(g)$ is the 
number of periodic points of period $m$. The Artin-Mazur zeta function $\zeta=\zeta_{g}$ of $(Y, g)$ is defined as the formal power series

$$
\zeta_{g}(t)=\exp \left(\sum_{m=1}^{\infty} \frac{N_{m}}{m} t^{m}\right) .
$$

Remark 3.2. Since there is a bijection between fixed points of $f^{n}$ and $\bar{f}^{n}$ by Remark 2.3, it is straightforward that Artin-Mazur zeta function of $(X, f)$ and that of $(\bar{X}, \bar{f})$ are the same. So to compute Artin-Mazur zeta function of a 1-solenoid, we concentrate on its presentation.

Smale [6] defined another zeta function, called Lefschetz zeta function or false zeta function, using Lefschetz number of a fixed point. The Lefschetz zeta function of a diffeomorphism on a compact manifold counts the periodic points 'algebraically'.

Let $M$ be a compact smooth manifold and $h: M \rightarrow M$ a differentiable map. If $p \in M$ is an isolated fixed point of $h$, then Lefschetz number of $p$ is

$$
L(p, h)=\operatorname{sign} \text { of } \operatorname{det}(I-D h(p))
$$

where $I$ is the identity map on $M$.

Since 1-solenoids and their presentations are defined as topological objects without differentiable structures, we need to modify the definition of Lefschetz number to use Lefschetz zeta function in 1-solenoids. We follow Smale's argument of orientation preserving/reversing.

Theorem 3.3 ([6]). Let $M$ and $h: M \rightarrow M$ be as above. For $p \in$ Fix $(h), L(p, h)=$ $(-1)^{u} \Delta$ where $u$ is the dimension of unstable manifold $W^{u}(p)$ of $h$ at $p$ and $\Delta=+1$ if $h$ preserves orientation on $W^{u}(p)$ and $\Delta=-1$ if $h$ reverses it.

Suppose that $(X, f)$ is a presentation of a 1-solenoid and that $p \in X$ is a fixed point of $f$. When $p$ is a branch point of $X$, we select a small neighborhood $U$ of $p$ such that $U=\cup I_{i}$ where $I_{i}$ is a 1 -simplex and $\left\{I_{i}-\{p\}\right\}$ is a disjoint set. Then we divide $U$ into two classes $U_{L}$, the set of 1-simplices in $U$ which are on the 'left' of $p$, and $U_{R}$, the set of 1-simplices on the 'right' of $p$. By Nonfolding Axiom, Flattening Axiom and Standing Assumption, if image of a 1-simplex $I \in U_{L}$ under $f$ includes a 1-simplex in $U_{\alpha}$, where $\alpha=L$ or $R$, then image of every 1-simplex in $U_{L}$ includes the same 1-simplex in $U_{\alpha}$, and image of every 1-simplex in $U_{R}$ under $f$ includes a 1-simplex in $U_{\beta}$ where $\beta=L$ or $R$ and $\alpha \neq \beta$. So the following definition is a natural adjustment of Lefschetz number to 1-solenoids: 
Definition 3.4. Let $(X, f)$ be a presentation of a 1-solenoid and that $p \in X$ a fixed point of $f$. If $p$ is a branch point of $X$, we define

$$
L(p, f)= \begin{cases}-1 & \text { if image of } U_{L} \text { under } f \text { includes a simplex in } U_{L} \\ 1 & \text { if images of } U_{L} \text { includes a simplex } U_{R}\end{cases}
$$

If $p$ is a nonbranch point, we define

$$
L(p, f)= \begin{cases}-1 & \text { if } f \text { preserves orientation on a small neighborhood of } p \\ 1 & \text { if } f \text { reverses it. }\end{cases}
$$

We imitate Smale's definition of Lefschetz zeta functions for diffeomorphisms on compact smooth manifolds to 1-solenoids.

Definition $3.5([6])$. Suppose that $(X, f)$ is a presentation of a 1 -solenoid and that $p \in X$ is a fixed point of $f$ with the corresponding Lefschetz number $L(p, f)$. The Lefschetz zeta function $\tilde{\zeta}_{f}$ of $(X, f)$ is defined as

$$
\tilde{\zeta}_{f}(t)=\exp \left(\sum_{m=1}^{\infty} \frac{L_{m}}{m} t^{m}\right) \text { where } L_{m}=\sum_{p \in \operatorname{Fix}\left(f^{m}\right)} L\left(p, f^{m}\right) .
$$

When a 1-solenoid is orientable, Artin-Mazur zeta function and Lefschetz zeta function of the solenoid have a simple relation. Smale [6] proved the same relation for Anosov diffeomorphisms on manifolds, and we follow his argument.

Proposition 3.6. If $(X, f)$ is an orientable presentation of a 1-solenoid, then

$$
\tilde{\zeta}_{f}(t)= \begin{cases}\frac{1}{\zeta_{f}(t)} & \text { if } f \text { is orientation preserving } \\ \frac{1}{\zeta_{f}(-t)} & \text { if } f \text { is orientation reversing. }\end{cases}
$$

Proof. If $f$ is orientation preserving, then Lefschetz number of any periodic point is -1 . So $L_{m}=-N_{m}$ and

$$
\tilde{\zeta}_{f}(t)=\exp \left(\sum_{m=1}^{\infty} \frac{L_{m}}{m} t^{m}\right)=\exp \left(\sum_{m=1}^{\infty} \frac{-N_{m}}{m} t^{m}\right)=\exp \left(-\sum_{m=1}^{\infty} \frac{N_{m}}{m} t^{m}\right)=\frac{1}{\zeta_{f}(t)} .
$$

If $f$ is orientation reversing, then $f^{2 m-1}$ is orientation reversing and $f^{2 m}$ orientation preserving for every $m \geq 1$. Hence $L_{m}=(-1)^{m+1} N_{m}$ and

$$
\tilde{\zeta}_{f}(t)=\exp \left(\sum_{m=1}^{\infty} \frac{(-1)^{m+1} N_{m}}{m} t^{m}\right)=\exp \left(-\sum_{m=1}^{\infty} \frac{N_{m}}{m}(-t)^{m}\right)=\frac{1}{\zeta_{f}(-t)} .
$$

We need a few lemmas to compute zeta functions of nonorientable 1-solenoids.

The following lemma is trivial from Linear Algebra: 
Lemma 3.7. Let $(X, f)$ be a nonorientable presentation of a 1-solenoid and $(\tilde{X}, \tilde{f})$ its orientable double cover with their corresponding adjacency matrices $\tilde{M}$ and $M$, respectively. For the square nonnegative integer matrices $M_{1}$ and $M_{2}$ given in Proposition 2.5, we let $D=M_{1}-M_{2}$. Then

$$
\operatorname{det}(I-t \tilde{M})=\operatorname{det}(I-t M) \cdot \operatorname{det}(I-t D) .
$$

Let us divide $X$ into two relatively disjoint parts $X_{+}$and $X_{-}$such that $\left.f\right|_{X_{+}}$ is orientation-preserving and $\left.f\right|_{X_{-}}$is orientation-reversing. Then $M_{1}$ and $M_{2}$ are matrices representing $\left.f\right|_{X_{+}}: X_{+} \rightarrow X$ and $\left.f\right|_{X_{-}}: X_{-} \rightarrow X$. Hence we can obtain the number of fixed points of $f$ from the trace of $M=M_{1}+M_{2}$ and sum of Lefschetz numbers of fixed points of $f$ from negative of the trace of $D$.

Because a branch point $b$ is a periodic point by Markov Axiom and it is included in different edges as a common boundary point, $b$ will appear several times in $\operatorname{tr}\left(M^{m}\right)$ and $\operatorname{tr}\left(D^{m}\right)$ as a periodic point in different edges. Thus we have to remove the extra part of $b$ from $\operatorname{tr}\left(M^{m}\right)$ and $\operatorname{tr}\left(D^{m}\right)$.

Remark 3.8. Suppose that $b$ is a branch point of $X$ with a period $m$. Then, by Standing Assumption, there is a small open neighborhood $U_{b}$ of $b$ such that $f^{m}\left(U_{b}\right)$ is homeomorphic to an open interval by Flattening Axiom. So there is a set of small 1-simplices $I_{i}$ in $X$ such that

(1) $U_{b}=\cup I_{i}$,

(2) $\left\{I_{i}-\{b\}\right\}$ is a disjoint set,

(3) each $I_{i}$ is a subset of an edge of $X$, and

(4) by Flattening Axiom, there are at most four and at least two 1-simplices $I_{b_{i}}$ in $\left\{I_{i}\right\}$ such that $I_{b_{i}}$ and $f^{m}\left(I_{b_{i}}\right)$ are contained in the same edge of $X$.

Let us denote $J_{b}=\left\{I_{b_{i}}\right\}$ and $n(b)=\# J_{b}$. Then $b$ is counted $n(b)$ times as a periodic point of period $m$ in $\operatorname{tr}\left(M^{m}\right)$. We will use $J_{b}$ and $n(b)$ to adjust extra part in $\operatorname{tr}\left(M^{m}\right)$.

Lemma 3.9. If $L\left(b, f^{p(b)}\right)=-1$, then $L\left(b, f^{k p(b)}\right)=-1$ and $L\left(f^{k}(b), f^{p(b)}\right)=-1$ for every natural number $k$. And if $L\left(b, f^{p(b)}\right)=1$, then $L\left(b, f^{k p(b)}\right)=(-1)^{k+1}$ and $L\left(f^{k}(b), f^{p(b)}\right)=1$.

Proof. Let $b$ be a branch point with the minimal period $p(b)$. If $L\left(b, f^{p(b)}\right)=-1$, then $f^{p(b)}$ and $f^{k p(b)}$, for every $k \geq 1$, map 'left-hand side' simplices of $b$ to a lefthand side simplex and 'right-hand side' simplices to a right-hand side simplex. And if $L\left(b, f^{p(b)}\right)=1, f^{p(b)}$ maps left-hand side simplices of $b$ to a right-hand side simplex and vice versa. But $f^{2 p(b)}$ maps left-hand side of $b$ to a left-hand side simplex and 
right-hand side to a right-hand side simplex.

Note that $f(b)$ is also a branch point with the minimal period $p(b)$. Then $f: U_{b} \rightarrow$ $U_{f(b)}$ and $f: U_{f^{p(b)}\left(U_{b}\right)} \rightarrow U_{f^{p(b)+1}\left(U_{b}\right)}$ have the same orientation preserving/reversing property. So $f^{p(b)}: U_{b} \rightarrow U_{f^{p(b)}\left(U_{b}\right)}$ and $f^{p(b)}: U_{f(b)} \rightarrow U_{f^{p(b)+1}\left(U_{b}\right)}$ have the same Lefschetz number at $b$ and $f(b)$.

Lemma 3.10. Suppose $I_{b_{i}}$ and $J_{b}$ are as above. Let $p(b)$ be the minimal period of $b$ and $m$ a period of $b$ so that $m=k p(b)$ for some natural number $k$. Define

$$
l_{m}\left(I_{b_{i}}\right)= \begin{cases}+1 & \text { if }\left.f^{m}\right|_{I_{b_{i}}} \text { is orientation preserving } \\ -1 & \text { if }\left.f^{m}\right|_{I_{b_{i}}} \text { is orientation reversing. }\end{cases}
$$

If $L\left(b, f^{m}\right)=-1$, then $\sum_{I_{b_{i}} \in J_{b}} l_{m}\left(I_{b_{i}}\right) \in\{0,1,2\}$ and $\sum_{I_{b_{i}} \in J_{b}} l_{m}\left(I_{b_{i}}\right)+n(b)=4$. If $L\left(b, f^{m}\right)=1$, then $\sum_{I_{b_{i}} \in I_{b}} l_{m}\left(I_{b_{i}}\right)=-2$ and $\sum_{I_{b_{i}} \in J_{b}} l_{m}\left(I_{b_{i}}\right)+n(b)=0$.

Proof. Let $U_{b}$ be the neighborhood of $b$ constructed in Remark 3.8, and $f^{m}\left(U_{b}\right) \subset$ $e_{1} \cup e_{2}$ where $e_{1}$ and $e_{2}$ are (possibly the same) edges of $X$. Then each $I_{b_{i}} \in J_{b}$ is the initial or the terminal part of $e_{1}$ or $e_{2}$.

We consider $L\left(b, f^{m}\right)=-1$ case first. If $J_{b}$ has four elements, then $e_{1} \neq e_{2}$ and $J_{b}=\left\{I_{1 i}, I_{1 t}, I_{2 i}, I_{2 t}\right\}$ where 1 and 2 represent the edges $I_{*}$ are included and $i$ and $t$ represent initial and terminal part, respectively. By Flattening Axiom and Markov Axiom, $f^{m}\left(I_{1 i}\right)=f^{m}\left(I_{1 t}\right) \subset e_{1}$ is either the initial part or the terminal part of $e_{1}$, and $f^{m}\left(I_{2 i}\right)=f^{m}\left(I_{2 t}\right) \subset e_{2}$ is also either the initial part or the terminal part of $e_{2}$. So if $f^{m}\left(I_{1 i}\right)=f^{m}\left(I_{1 t}\right)$ is the initial part of $e_{1}$, then $\left.f^{m}\right|_{I_{1 i}}$ is orientation preserving and $\left.f^{m}\right|_{I_{1 t}}$ is orientation reversing. And if $f^{m}\left(I_{1 i}\right)=f^{m}\left(I_{1 t}\right)$ is the terminal part of $e_{1}$, then we will have opposite result for $\left.f^{m}\right|_{I_{1 *}}$. Hence we have $\sum_{I_{*} \in J_{b}} l_{m}\left(I_{*}\right)=0$.

If $J_{b}$ has three elements, then $e_{1} \neq e_{2}$ and $J_{b}=\left\{I_{1 i}, I_{1 t}, I_{2}\right\}$ such that $f^{m}\left(I_{1 i}\right)=$ $f^{m}\left(I_{1 t}\right)$. Thus, as in the above case, $\left.f^{m}\right|_{I_{1 i}}$ is orientation preserving and $\left.f^{m}\right|_{I_{1 t}}$ is orientation reversing, or vice versa. And by Expansion Axiom and Flattening Axiom, $f^{m}\left(I_{2}\right)$ includes $I_{2}$ so that $\left.f^{m}\right|_{I_{2}}$ is orientation preserving. Thence we have $\sum_{I_{*} \in J_{b}} l_{m}\left(I_{*}\right)=1$.

If $J_{b}$ has two elements $I_{1}$ and $I_{2}$, then by Expansion and Flattening Axioms $f^{m}\left(I_{*}\right)$ includes $I_{*}$. So $\left.f^{m}\right|_{I_{*}}$ is orientation preserving, and $\sum_{I_{*} \in J_{b}} l_{m}\left(I_{*}\right)=2$.

Let $L\left(b, f^{m}\right)=1$ and $f^{m}\left(U_{b}\right) \subset e_{1} \cup e_{2}$. Assume $I_{1 i}, I_{1 t} \in J_{b}$. Then there are two cases: Both of $I_{1 i}$ and $I_{1 t}$ are included in the same side of $b$, say left-hand side. Or one is on the left-hand side of $b$ and the other is on the right-hand side.

If $I_{1 i}$ and $I_{1 t}$ are included in the left-hand side, then $L\left(b, f^{m}\right)=1$ implies that $f^{m}\left(I_{1 *}\right)$ is mapped to the right-hand side. So $f^{m}\left(I_{1 *}\right)$ is not included in $e_{1}$, and 
$I_{1 i}, I_{1 t} \notin J_{b}$. Hence we conclude that $I_{1 i}, I_{1 t} \in J_{b}$ implies $I_{1 t} \subset f^{m}\left(I_{1 i}\right)$ and $I_{1 i} \subset$ $f^{m}\left(I_{1 t}\right)$ so that $f^{m}\left(U_{b}\right)$ is a subset of $e_{1}$ and $J_{b}=\left\{I_{1 i}, I_{1 t}\right\}$.

If $J_{b}$ has more than two elements, then two of them are included in the same edge of $X$ and any remaining simplex cannot be an element of $J_{b}$. Therefore, when $L\left(b, f^{m}\right)=1, J_{b}$ has two elements $I_{1}, I_{2}$. And it is obvious that $\left.f^{m}\right|_{I_{*}}$ is orientation reversing as $L\left(b, f^{m}\right)=1$.

Combining Lemmas 3.9 and 3.10, we have the following: Let $p(b)$ be the minimal period of $b$. If $L\left(b, f^{p(b)}\right)=-1$, then $f^{k p(b)}$ is orientation preserving at $b$ for every $k \geq 1$ so that

$$
\sum l_{p(b)}\left(I_{b_{i}}\right)=\sum l_{k p(b)}\left(I_{b_{i}}\right) \in\{0,1,2\} .
$$

And if $L\left(b, f^{p(b)}\right)=1$, then $f^{(2 k-1) p(b)}$ is orientation reversing and $f^{2 k p(b)}$ is orientation preserving at $b$ for every $k \geq 1$ so that

$$
\begin{aligned}
\sum l_{p(b)}\left(I_{b_{i}}\right) & =\sum l_{(2 k-1) p(b)}\left(I_{b_{i}}\right)=-2 \text { and } \\
\sum l_{2 p(b)}\left(I_{b_{i}}\right) & =\sum l_{2 k p(b)}\left(I_{b_{i}}\right) \in\{0,1,2\} .
\end{aligned}
$$

Let us denote $s_{1}(b)=\sum l_{p(b)}\left(I_{b_{i}}\right)$ and $s_{2}(b)=\sum l_{2 p(b)}\left(I_{b_{i}}\right)$.

Proposition 3.11. Suppose that $(X, f)$ is a nonorientable presentation of a 1solenoid and $(\tilde{X}, \tilde{f})$ is its orientable double cover. Then Artin-Mazur zeta function of $(\tilde{X}, \tilde{f})$ is given by

$$
\zeta_{\tilde{f}}(t)=\frac{\zeta_{f}(t)}{\tilde{\zeta}_{f}(t)}
$$

Proof. We compute $\tilde{\zeta}_{f}(t), \zeta_{f}(t)$ and $\zeta_{\tilde{f}}(t)$ separately to show the quotient relation among them.

$\tilde{\zeta}_{f}(t)$ : Let $D=M_{1}-M_{2}$ be the matrix in Lemma 3.7. If $p$ is a nonbranch fixed point of $f^{m}$ and $I$ is a small neighborhood of $p$ properly contained in an edge of $X$, then, on the diagonal of $D^{m}, p$ is counted +1 when $f^{m}$ is orientation preserving on $I$ and -1 when $f^{m}$ is orientation reversing on $I$. Hence Lefschetz number of each nonbranch fixed point of $f^{m}$ is counted once in $-\operatorname{tr}\left(D^{m}\right)$.

Suppose that $b$ is a branch point of $X$ with a period $m$ and that $J_{b}=\left\{I_{b_{i}}\right\}$ is as in Remark 3.8. Since $b$ is a common boundary point of $I_{b_{1}} \subset e_{b_{1}}$ and $I_{b_{2}} \subset e_{b_{2}}, b$ is counted $\sum_{I_{b_{i}} \in J_{b}} l_{m}\left(I_{b_{i}}\right)$ times in $\operatorname{tr}\left(D^{m}\right)$ instead of $-L\left(b, f^{m}\right)$. Thus

$$
L_{m}=\sum_{p \in \operatorname{Fix}\left(f^{m}\right)} L\left(p, f^{m}\right)=-\left\{\operatorname{tr}\left(D^{m}\right)-\sum_{b}\left(\sum l_{m}\left(I_{b_{i}}\right)+L\left(b, f^{m}\right)\right)\right\} \text {. }
$$


Then by Lemma 3.9 we have

$$
\begin{aligned}
& L_{m}=\sum_{p \in \operatorname{Fix}\left(f^{m}\right)} L\left(p, f^{m}\right) \\
& =-\operatorname{tr}\left(D^{m}\right) \\
& +\underbrace{\sum_{b} s_{1}(b)+L\left(b, f^{p(b)}\right)}_{\substack{p(b) \text { divides } m \text { and } \\
L\left(b, f^{p(b)}\right)=-1}}+\underbrace{\sum_{b} s_{1}(b)+L\left(b, f^{p(b)}\right)}_{\begin{array}{c}
m=(2 k-1) p(b) \text { and } \\
L\left(b, f^{p(b)}\right)=1
\end{array}}+\underbrace{\sum_{b} s_{2}(b)+L\left(b, f^{2 p(b)}\right)}_{\begin{array}{c}
m=2 k p(b) \text { and } \\
L\left(b, f^{p(b)}\right)=1
\end{array}} \\
& =-\operatorname{tr}\left(D^{m}\right)+\underbrace{\sum s_{1}(b)-1}_{\substack{p(b) \text { divides } m \text { and } \\
L\left(b, f^{p(b)}\right)=-1}}+\underbrace{\sum s_{1}(b)+1}_{\substack{m=(2 k-1) p(b) \text { and } \\
L\left(b, f^{p(b)}\right)=1}}+\underbrace{\sum s_{2}(b)-1}_{\begin{array}{c}
m=2 k p(b) \text { and } \\
L\left(b, f^{p(b)}\right)=1
\end{array}}
\end{aligned}
$$

and

$$
\begin{aligned}
\tilde{\zeta}_{f}(t)= & \exp \left(\sum_{m=1}^{\infty} \frac{L_{m}}{m} t^{m}\right) \\
= & \operatorname{det}(I-t D) \\
& \cdot \frac{1}{\prod_{L(b)=-1}\left(1-t^{p(b)}\right)^{\frac{s_{1}(b)-1}{p(b)}}} \cdot \frac{\prod_{L(b)=1}\left(1+t^{p(b)}\right)^{\frac{s_{1}(b)+1}{2 p(b)}}}{\prod_{L(b)=1}\left(1-t^{p(b)}\right)^{\frac{s_{1}(b)+1}{2 p(b)}}} \cdot \frac{1}{\prod_{L(b)=1}\left(1-t^{2 p(b)}\right)^{\frac{s_{2}(b)-1}{2 p(b)}}} .
\end{aligned}
$$

$\zeta_{f}(t)$ : Let $M$ be the adjacency matrix of $(X, f)$ and $J_{b}$ as in Remark 3.8 for a branch point $b$ of $X$. We remind that $J_{b}$ with $n(b)=\# J_{b}$ is the collection of small 1-simplices $I_{i}$ containing $b$ as their boundary point such that $I_{i}$ and $f^{p(b)}\left(I_{i}\right)$ are included in a same edge of $X$.

In $\operatorname{tr}\left(M^{m}\right)$, every nonbranch periodic point of period $m$ is counted once. But a branch point $b$ of period $m$, which is an intersection of several different edges of $X$, is counted $n(b)$ times in $\operatorname{tr}\left(M^{m}\right)$. Hence if we subtract $n(b)-1$ from $\operatorname{tr}\left(M^{m}\right)$, then $b$ will be counted once as a periodic point of period $m$. Therefore

$$
\begin{aligned}
N_{m}= & \operatorname{tr}\left(M^{m}\right)-\sum_{\substack{p(b) \mid m \text { and } \\
L(b)=-1}}(n(b)-1) \\
& -\sum_{\substack{m=(2 n-1) p(b) \\
\text { and } L(b)=1}}(n(b)-1)-\sum_{\substack{m=2 n p(b) \\
\text { and } L(b)=1}}(n(b)-1)
\end{aligned}
$$


and

$$
\begin{aligned}
\zeta_{f}(t) & =\exp \left(\sum_{m=1}^{\infty} \frac{N_{m}}{m} t^{m}\right) \\
& =\frac{\prod_{L(b)=-1}\left(1-t^{p(b)}\right)^{\frac{n(b)-1}{p(b)}} \cdot \prod_{L(b)=1}\left(1-t^{p(b)}\right)^{\frac{n(b)-1}{2 p(b)}} \cdot \prod_{L(b)=1}\left(1-t^{2 p(b)}\right)^{\frac{n(b)-1}{2 p(b)}}}{\operatorname{det}(I-t M) \cdot \prod_{L(b)=1}\left(1+t^{p(b)}\right)^{\frac{n(b)-1}{2 p(b)}}} .
\end{aligned}
$$

$\zeta_{\tilde{f}}(t)$ : As in the case of $\operatorname{tr}\left(M^{m}\right)$, we have to remove over-counted parts of branch points in $\tilde{X}$ from $\operatorname{tr}\left(\tilde{M}^{m}\right)$. We remind that a point in $\tilde{X}$ is a branch point if and only if it is a fiber of a branch point in $X$.

Let $b$ be a branch point of $X$ with its fibers $b_{1}, b_{2}$ in $\tilde{X}$. Then orientability of $(\tilde{X}, \tilde{f})$ and Flattening Axiom imply $n\left(b_{i}\right)=\# J_{b_{i}}=2$ so that $b_{i}$ is counted twice as a fixed point in $\tilde{M}^{k p\left(b_{i}\right)}$. Hence we only need to know what is $p\left(b_{i}\right)$. And it is straightforward from the construction of $(\tilde{X}, \tilde{f})$, referring [11], that

(1) $p\left(b_{i}\right)=p(b)$ if $L\left(b, f^{p(b)}\right)=-1$

(2) $p\left(b_{i}\right)=2 p(b)$ if $L\left(b, f^{p(b)}\right)=1$.

Thus the number of periodic points of period $m$ in $(\tilde{X}, \tilde{f})$ is

$$
\begin{aligned}
\tilde{N}_{m} & =\operatorname{tr}\left(\tilde{M}^{m}\right)-\sum_{\substack{p\left(b_{i}\right) \mid m \text { and } \\
L(b)=-1}}(2-1)-\sum_{\substack{p\left(b_{i}\right) \mid m \text { and } \\
L(b)=1}}(2-1) \\
& =\operatorname{tr}\left(\tilde{M}^{m}\right)-\sum_{\substack{p(b) \mid m \text { and } \\
L(b)=-1}} 2(2-1)-\sum_{\substack{2 p(b) \mid m \text { and } \\
L(b)=1}} 2(2-1)
\end{aligned}
$$

and zeta function of $(\tilde{X}, \tilde{f})$ is

$$
\zeta_{\tilde{f}}(t)=\exp \left(\frac{\tilde{N}_{m}}{m} t^{m}\right)=\frac{\prod_{L(b)=-1}\left(1-t^{p(b)}\right)^{\frac{2(2-1)}{p(b)}} \cdot \prod_{L(b)=1}\left(1-t^{2 p(b)}\right)^{\frac{2(2-1)}{2 p(b)}}}{\operatorname{det}(I-t \tilde{M})} .
$$

Recall that by Lemma 3.10

(1) if $L\left(b, f^{p(b)}\right)=-1$, then $s_{1}(b)+n(b)=4$

(2) if $L\left(b, f^{p(b)}\right)=1$, then $s_{1}(b)+n(b)=0$ and $s_{2}(b)+n(b)=4$

And by Lemma 3.7

$$
\operatorname{det}(I-t \tilde{M})=\operatorname{det}(I-t M) \cdot \operatorname{det}(I-t D) .
$$


Therefore

$$
\begin{aligned}
& \zeta_{\tilde{f}}(t)=\frac{\prod_{L(b)=-1}\left(1-t^{p(b)}\right)^{\frac{2(2-1)}{p(b)}} \cdot \prod_{L(b)=1}\left(1-t^{2 p(b)}\right)^{\frac{2(2-1)}{2 p(b)}}}{\operatorname{det}(I-t \tilde{M})} \\
& =\frac{\prod_{L(b)=-1}\left(1-t^{p(b)}\right)^{\frac{s_{1}(b)+n(b)-2}{p(b)}} \cdot \prod_{L(b)=1}\left(1-t^{2 p(b)}\right)^{\frac{s_{2}(b)+n(b)-2}{2 p(b)}}}{\operatorname{det}(I-t M) \cdot \operatorname{det}(I-t D)} \\
& =\frac{\prod_{L(b)=-1}\left(1-t^{p(b)}\right)^{\frac{s_{1}(b)+n(b)-2}{p(b)}} \cdot \prod_{L(b)=1}\left(1-t^{2 p(b)}\right)^{\frac{s_{2}(b)+n(b)-2}{2 p(b)}}}{\operatorname{det}(I-t M) \cdot \operatorname{det}(I-t D)} \\
& \frac{\prod_{L(b)=1}\left(1-t^{p(b)}\right)^{\frac{s_{1}(b)+n(b)}{2 p(b)}}}{\prod_{L(b)=1}\left(1+t^{p(b)}\right)^{\frac{s_{1}(b)+n(b)}{2 p(b)}}} \\
& =\frac{\prod_{L(b)=-1}\left(1-t^{p(b)}\right)^{\frac{n(b)-1}{p(b)}} \cdot \prod_{L(b)=1}\left(1-t^{2 p(b)}\right)^{\frac{n(b)-1}{2 p(b)}} \cdot \prod_{L(b)=1}\left(1-t^{p(b)}\right)^{\frac{n(b)-1}{2 p(b)}}}{\operatorname{det}(I-t M) \cdot \prod_{L(b)=1}\left(1+t^{p(b)}\right)^{\frac{n(b)-1}{2 p(b)}}} \\
& \cdot \frac{\prod_{L(b)=-1}\left(1-t^{p(b)}\right)^{\frac{s_{1}(b)-1}{p(b)}} \cdot \prod_{L(b)=1}\left(1-t^{2 p(b)}\right)^{\frac{s_{2}(b)-1}{2 p(b)}} \cdot \prod_{L(b)=1}\left(1-t^{p(b)}\right)^{\frac{s_{1}(b)+1}{2 p(b)}}}{\operatorname{det}(I-t D) \cdot \prod_{L(b)=1}\left(1+t^{p(b)}\right)^{\frac{s_{1}(b)+1}{2 p(b)}}} \\
& =\zeta_{f}(t) \cdot \frac{1}{\tilde{\zeta}_{f}(t)} .
\end{aligned}
$$

Remark 3.12. Franks [4] showed similar results for an Axiom A diffeomorphism on a manifold.

Proposition 3.13. Suppose that $(X, f)$ and $(\tilde{X}, \tilde{f})$ are as above. Then $\zeta_{\tilde{f}}(t)$ is a rational function.

Proof. To show that $\zeta_{\tilde{f}}(t)$ is a rational function, we look at the orbit of a branch point and the numerator of $\zeta_{\tilde{f}}(t)$,

$$
\prod_{L(b)=-1}\left(1-t^{p(b)}\right)^{\frac{2(2-1)}{p(b)}} \cdot \prod_{L(b)=1}\left(1-t^{2 p(b)}\right)^{\frac{2(2-1)}{2 p(b)}} .
$$


Let $b$ be a branch point with the minimal period $p(b)$ and $L(b)=1$. Then, for $1 \leq k<p(b), f^{k}(b)$ is also a branch point with the minimal period $p(b)$ and $L\left(f^{k}(b)\right)=L(b)$ by Lemma 3.9. So $\left(1-t^{p(b)}\right)^{\frac{2(2-1)}{p(b)}}=\left(1-t^{p\left(f^{k}(b)\right)}\right)^{\frac{2(2-1)}{p\left(f^{k}(b)\right)}}$ implies that there are $p(b)$ copies of $\left(1-t^{p(b)}\right)^{\frac{2(2-1)}{p(b)}}$ inside the first product of $(1)$. Hence the first product is equal to

$$
\prod_{O(b) \text { with } L(b)=-1}\left(1-t^{p(b)}\right)^{2(2-1)}
$$

where $O(b)$ is the orbit of $b$. And by the same argument, the second product of (1) is equal to

$$
\prod_{O(b) \text { with } L(b)=1}\left(1-t^{2 p(b)}\right)^{(2-1)}
$$

Therefore the numerator of Artin-Mazur zeta function of $(\tilde{X}, \tilde{f})$ is a polynomial, and it is a rational function.

Corollary 3.14. Let $(X, f)$ and $(\tilde{X}, \tilde{f})$ be as above. Then Lefschetz zeta function of $(\tilde{X}, \tilde{f})$ is a rational function given by

$$
\tilde{\zeta}_{\tilde{f}}(t)=\frac{\tilde{\zeta}_{f}(-t)}{\zeta_{f}(-t)}
$$

Proof. Since $(\tilde{X}, \tilde{f})$ is orientable, $\tilde{\zeta}_{\tilde{f}}(t)=1 / \zeta_{\tilde{f}}(-t)$ by Proposition 3.6. Then it is trivial by Proposition 3.11.

Example 3.15. Suppose that $(X, f)$ and $(\tilde{X}, \tilde{f})$ are as in Example 2.6. Then two branch points $b_{1}, b_{2}$ of $X$ are periodic points of minimal period 2. For $i=1,2$, we have $L\left(b_{i}, f^{2 m}\right)=-1$ from the wrapping rule, $s_{i}\left(b_{i}\right)=1$ from the fact that $f^{2}$ is orientation preserving, and $n\left(b_{i}\right)=3$ as each $b_{i}$ is the vertex of Y-shaped simplex. Hence Artin-Mazur zeta function and Lefschetz zeta function for $(X, f)$ and Artin-Mazur zeta function for $(\tilde{X}, \tilde{f})$ are

$$
\zeta_{f}(t)=\frac{1-t^{2}}{1-3 t}, \quad \tilde{\zeta}_{f}(t)=(1-t)\left(1-t^{2}\right), \text { and } \zeta_{\tilde{f}}(t)=\frac{1}{(1-3 t)(1-t)} .
$$

\section{REFERENCES}

1. M. Artin \& B. Mazur: On periodic points. Ann. of Math. 81 (1965), 82-99.

2. A. Fel'shtyn: Dynamical zeta functions, Nielsen theory and Reidemeister torsion. Mem. Amer. Math. Soc. 147 (2000), no. 699. 
3. R. Fokkink: The structure of trajectories. Ph.D. Thesis, Technische Universiteit te Delft, 1991.

4. J. Franks: A reduced zeta functions for diffeomorphisms. Amer. J. Math. 100 (1978), 217-243.

5. D. Ruelle: Dynamical zeta functions for piecewise monotone maps of the interval. CRM Monograph Series 4, Amer. Math. Society, 1994.

6. S. Smale: Differentiable dynamical systems. Bull. Amer. Math. Soc. 73 (1967), 747-817.

7. R.F. Williams: One-dimensional non-wandering sets. Topology 6 (1967), 473-487.

8. _ Classification of 1-dimensional attractors. Proc. Symp. Pure Math. 14 (1970), 341-361.

9. __ Expanding attractors. IHES Publ. Math. 43 (1974), 169-203.

10. I. Yi: Canonical symbolic dynamics for one-dimensional generalized solenoids. Trans. Amer. Math. Soc. 353 (2001), 3741-3767.

11. _ _ : Ordered group invariants for nonorientable one-dimensional generalized solenoids. Proc. Amer. Math. Soc. 131 (2003), 1273-1282.

12. A.Yu. Zhirov: Hyperbolic attractors of diffeomorphisms of orientable surfaces. Russian Acad. Sci. Sb. Math. 82 (1995), 135-174.

Department of Mathematics Education, Ewha Womans University, Seoul 120-750, KoREA

Email address: yih@ewha.ac.kr 